\title{
Annotating Geographical Objects in OpenStreetMap with Geo-tagged Social Media
}

\author{
Xin Chen Hoang Vo Fusheng Wang \\ Stony Brook University \\ \{xin.chen.1,hvvo,fusheng.wang\}@stonybrook.edu
}

\begin{abstract}
Recent years have witnessed an explosion of geospatial data, especially in the form of Volunteered Geographic Information (VGI). As a prominent example, OpenStreetMap (OSM) creates a free editable map of the world from a large number of contributors. On the other hand, social media platforms such as Twitter or Instagram supply dynamic social feeds at population level. As much of such data is geo-tagged, there is a high potential on integrating social media with OSM to enrich OSM with semantic annotations, which will complement existing objective description oriented annotations to provide a broader range of annotations. In this paper, we propose a comprehensive framework on integrating social media data and VGI data to derive knowledge about geographical objects, specifically, top relevant annotations from tweets for objects in OSM. We first integrate geo-tagged tweets with OSM data with scalable spatial queries running on MapReduce. We propose a frequency based method for annotating boundary based geographic objects, and a probability based method for annotating point based geographic objects, with consideration of noise. We evaluate our methods using a large geo-tagged tweets corpus and representative geographic objects from OSM, which demonstrates promising results through ground-truth comparison and case studies. We are able to produce up to $80 \%$ correct names for geographical objects and discover implicitly relevant information, such as popular exhibitions of a museum, the nicknames or visitors' impression to a tourism attraction.
\end{abstract}

\section{INTRODUCTION}

Large scale Volunteered Geographical Information (VGI)[5], such as OpenStreetMap (OSM) and Wikimapia, has created high potential for establishing reliable geographical information. As a prominent example, OSM accelerates the generation of massive geospatial information from community users and currently has more than 3.7 billion geographical objects. OSM is not only used by end users, but also adopted by companies to support many geospatial services.

Permission to make digital or hard copies of all or part of this work for personal or classroom use is granted without fee provided that copies are not made or distributed for profit or commercial advantage and that copies bear this notice and the full citation on the first page. Copyrights for components of this work owned by others than the author(s) must be honored. Abstracting with credit is permitted. To copy otherwise, or republish, to post on servers or to redistribute to lists, requires prior specific permission and/or a fee. Request permissions from permissions@acm.org.

ACM SIGSPATIA LBSN 16, October 31-November 03 2016, Burlingame, CA, USA

(C) 2016 Copyright held by the owner/author(s). Publication rights licensed to ACM. ISBN $978-1-4503-4586-6 / 16 / 10 \ldots \$ 15.00$

DOI: http://dx.doi.org/10.1145/3021304.3021306
OSM aims to provide two types of information about geographical objects: 1) geographical boundaries such as points, lines and regions and 2) annotations or tags. A tag consists of a 'Key' and a 'Value' to describe the objects. Example objects include building footprints, business places, or tourist attractions. The keys provide a broad class of features (e.g., building or amenity) while the values detail the specific features, e.g., "building=retail" or "amenity=school".

On the other hand, much of the social media data is associated with geo-locations. A recent study ${ }^{1}$ shows that $1.0 \%$ of tweets are geotagged, and $87 \%$ of geotagged tweets contain exact coordinates. This shows a major increase of geotagged tweets from $0.23 \%$ shown in a study in $2010^{2}$. Such geo-tagged tweets, if combined, could provide rich information that can be potentially associated with other geospatial data. For example, the work in [12] uses geo-tagged tweets as external contextual data to annotate mobile users. One natural question is, can we use such geo-tagged social media to support semantic annotations for geographical objects such as churches, museums, and tourism attractions?

\begin{tabular}{|c|c|c|}
\hline OSM ID & Spatial Attribute & Name of the Place (from Existing OSM Tags) \\
\hline 4268203 & MULTIPOLYGON(...) & The All England Lawn Tennis Club \\
\hline 37118074 & MULTIPOLYGON(...) & Stonehenge \\
\hline 253427773 & POINT (-6.5202424 55.201863) & Old Bushmills Distillery \\
\hline \multicolumn{3}{|c|}{ Input: geo-tagged tweets } \\
\hline Tweet ID & Spatial Attribute & Tweet Content \\
\hline $61948 * * *$ & POINT (-0.2159 51.4355) & $\begin{array}{l}\text { Big screen for Murray vs Federer. Having an } \\
\text { awesome day. Wimbledon } 2015 .\end{array}$ \\
\hline $6138^{* * * *}$ & POINT (-1.8262 51.1789) & $\begin{array}{l}\text { Late for the Solstice.. (@ Stonehenge - } \\
\text { @englishheritage in Amesbury, Wiltshire) }\end{array}$ \\
\hline $63036^{* * *}$ & POINT (-6.5199 55.2019) & $\begin{array}{l}\text { oldest whiskey distillery in the world ... cant } \\
\text { believe Id never been! \#bushmills \#distillery }\end{array}$ \\
\hline \multicolumn{3}{|c|}{ Output: Annotations for OSM objects } \\
\hline Place ID & & Annotations \\
\hline 4268203 & \multicolumn{2}{|c|}{ wimbledon, tennis, lawn, murray, federer, henman, mound, djokovic, aeltc, ... } \\
\hline 37118074 & \multicolumn{2}{|c|}{ stonehenge, amesbury, wiltshire, rock, ancient, history, solstice, ... } \\
\hline 253427773 & \multicolumn{2}{|c|}{ bushmills, distillery, causeway, whiskey, NorthernIreland, oldest, giant, ... } \\
\hline
\end{tabular}

Figure 1: Examples that use geo-tagged tweet to annotate geographical objects in OSM.

In this paper, we propose to enrich OSM objects with semantic annotations by integrating and analyzing geo-tagged social media data. This will complement OSM's objective description oriented annotations to provide a broader range of annotations. Thus, it could significantly improve the

\footnotetext{
1 https://www.quora.com/What-percentage-of-tweets-are-geotagged-Whatpercentage-of-geotagged-tweets-are-ascribed-to-a-venue

${ }^{2}$ http://thenextweb.com/2010/01/15/twitter-geofail-023-tweetsgeotagged
} 
value of OSM to support geospatial services. Figure 1 illustrates the process for annotating OSM with geo-tagged tweets. For example, the All-England Club (best known as the venue for the Wimbledon Championships) is annotated with the acronym (aeltc), the nicknames (henman hill), and the players' names (murray, federer, djokovic). The Stonehenge (a famous prehistoric monument in Wiltshire, England) is annoated with the summer solstice (a popular festival hosting at Stonehenge).

We formalize the problem as to find a ranking function that could rank relevant social signals on top of non-relevant ones. Different from traditional information retrieval problems, a new spatial context is introduced into the problem. Thus, our approach will capture both the relevance and the locality of annotation candidates given a targeted location. As described next, major challenges exist for such spatial semantic annotation problem.

One immediate challenge is the difficulty with the estimation of spatial locality due to the diversity of geographical object representations. In OSM, many objects are represented with boundaries, for example, polygons. However, many objects only have a simple point based representation due to limited information or due to the small extent of the objects. For example, less than half of churches in OSM have boundaries ${ }^{3}$.

We propose two alternative methods that handle the two types of spatial objects: frequency based methods for objects with a clear boundary, and probability based method for objects with a point based representation. For the frequency based method, we consider all the tweets contained in the boundary of an object for the analysis. For probability based method, we estimate the probability of a nearby tweet for annotation contribution with respect to the distance between the tweet and the object.

Another major challenge is the noisy feature of social media data. Social media comprise a broad range of topics. Social media contain large amount of informal languages and personal trivial words from interpersonal chatting, which requires carefully tuned methods to extract meaningful semantic information.

We provide multiple approaches to reduce or remove the effect of noises from signals. For frequency based methods, we provide multiple ways to weigh the relevance of terms for objects. For probability based method with Kernel Density Estimation (KDE), we provide an adaptive approach to minimize the noise effect by tuning the kernel bandwidth inversely with the word density.

While it is difficult to provide ground-truth to evaluate semantic annotations, we propose two alternative approaches to validate our work. We first validate the explicitly relevant annotations with names of places, for which the ground truth is available, and then propose to validate our methods with case studies, with manual evaluation of the relevance of annotation words.

The paper is organized as follows. We first define the problem and provide an overview of methods in Section 2. We then present frequency based methods in Section $\mathbf{3}$, and probability based method in Section 4. We evaluate our methods using a large geo-tagged tweets corpus and representative OSM objects from in Section 5, followed by related work and conclusion.

\footnotetext{
$\overline{{ }^{3} \text { http://taginfo.openstreetmap.org/ }}$
}

\section{OVERVIEW}

\subsection{Problem Definition}

Our goal is to use geo-tagged social media to annotate geographical objects. We first define our problem as follows.

\subsubsection{Geographic Objects}

VGI platforms, such as OSM or Wikipedia, contain a large amount of geographic objects. In our problem setting, we consider two common representations of geographic objects: 1) point based geographic objects and 2) boundary based geographic objects. Point based geographic objects are a set of points $\mathbf{P}=\left\{p_{1}, p_{2}, \ldots, p_{N_{P}}\right\}$ where each object $p_{i}$ $=\left[i d_{p_{i}}, l_{p_{i}}\right]$ is represented as a single point in space with an object ID $i d_{p_{i}}$ and the location (latitude, longitude): $l_{p_{i}}=\left(x_{l_{p}}, y_{l_{p}}\right)$. Boundary based geographic objects are a set of polygons $\mathbf{B}=\left\{b_{1}, b_{2}, \ldots, b_{N_{B}}\right\}$ where each object $b_{i}$ $=\left[i d_{b}, L_{b}\right]$ is represented with an object ID and a closed polygon consisting of an ordered list of points that delineates the boundary of the object in space. The boundary $L_{b}=\left\{l_{1}, l_{2}, \ldots, l_{N}\right\}$ consists of latitude and longitude based point $l_{i}=\left(x_{l_{i}}, y_{l_{i}}\right)$.

\subsubsection{Geo-tagged Social Media}

The geo-tagged social media signals can be represented as a set of documents $\mathbf{D}=\left\{d_{1}, d_{2}, \ldots, d_{N_{D}}\right\}$. Each document $d_{j}$ for $j=1,2, \ldots, N_{D}$ consists a tuple $<i d_{d_{j}}, i d_{u}, l_{d_{j}}, W_{d_{j}}>$ where $i d_{d_{j}}$ and $i d_{u}$ denotes the ID of the document and the ID of the user who generates this content. The document location $l_{d_{j}}$ is a single point in the space represented by latitude and longitude based position $\left(x_{d_{j}}, y_{d_{j}}\right)$. $\mathbf{W}_{\mathbf{d}_{\mathbf{j}}}=$ $\left\{w_{1}, w_{2}, \ldots, w_{N_{W}}\right\}$ indicates a set of associated features extracted from the document $d_{j}$. While social media provide a wide range of signals such as image, video, their associated tags or metadata, for our study, we focus on uni-grams from tweet content.

\subsubsection{The Semantic Annotation Problem}

Given a collection of geographic objects $\mathbf{P}$ or $\mathbf{B}$ and a spatial-sensitive social media corpus $\mathbf{D}$, our goal is to integrate geographic information in $\mathbf{P}$ and $\mathbf{B}$ and social contextual information in $\mathbf{D}$. With integrated geospatial data, this work focuses on extracting semantic annotations from social signals w.r.t. fine-grained geographic objects either in the form of a point $\mathbf{P}$ or a polygon $\mathbf{B}$. For example, restaurants or coffee shops are typically represented as points, and building footprints, parking lots, or pitches are represented as polygons. With a variety of scales, churches, on the other hand, have no dominant form for spatial representations. The semantic annotations for a targeted geographic object is a set of relevant words, $\mathbf{A}=\left\{\left(w_{1}, s_{1}\right),\left(w_{2}, s_{2}\right), \ldots,\left(w_{N_{A}}, s_{N_{A}}\right)\right\}$, where $s_{i}\left(i=1,2, \ldots, N_{A}\right)$ is a score that measures the relevance of $w_{i}$ w.r.t. the geographic object.

The semantic annotation problem can be defined as to find a ranking function $\mathbf{f}\left(\mathbf{p}_{\mathbf{i}}, \mathbf{w}_{\mathbf{j}}\right)$ (or $\left.\mathbf{f}\left(\mathbf{b}_{\mathbf{i}}, \mathbf{w}_{\mathbf{j}}\right)\right)$ for a word $w_{i} \epsilon V_{D}$ w.r.t. a given geographic object $p_{i}$ or $b_{i}$, where $\mathbf{V}_{\mathbf{D}}=\left\{w_{1}, w_{2}, \ldots, w_{N}\right\}$ refers to the vocabulary that includes all annotation candidates generated from the social media corpus $D$. Analogous to a typical information retrieval task, our goal is to provide satisfactory ranking function to rank relevant annotation keywords on top of nonrelevant ones. 


\subsection{Overview of Methods}

The key challenge of the spatial semantic annotation problem is how to measure the likelihood that a word $w_{j}$ is relevant to the given geographic object. A unique constraint for our problem is that the annotation candidates and the annotating targets possess a spatial context, whereas a traditional information retrieval problem ranks relevant documents to a query. Thus, our goal is to propose an applicable model that should capture both the relevance and the locality of annotation words w.r.t. the targeted locations of geographic objects.

\subsubsection{Spatial Data Integration}

The spatial relevance of tweets will be largely affected by the proximity to the geographical objects, the extent and representation of the geographical objects. For a boundary based geographic object, intuitively, tweets contained in the boundary will likely have a higher "signal to noise ratio" than those outside of the boundary. For point based geographic objects, similarly, nearby tweets within a distance should have higher relevance than those outside, and the relevance could be affected by the distance.

To capture the spatial locality of words, we propose to use spatial queries to cross-match tweets with geographical objects, and filter tweets based on spatial proximity. Due to the massive volume of geospatial objects and the high computational complexity associated spatial queries, we first perform skew-aware space partitioning to generate balanced tiles, and then run spatial queries for each title in parallel through MapReduce by invoking an on-demand spatial query engine. We then normalize query results for objects across boundaries. We extend our current work HadoopGIS[1, 2, 11] to support the queries needed for such data integration. The two query scenarios are illustrated in Figure 2.

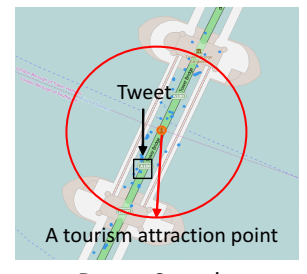

Range Search

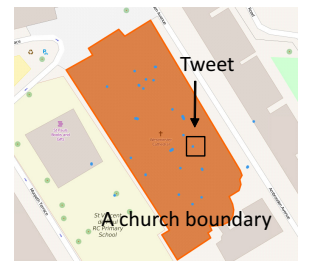

POINT-IN-POLYGON
Figure 2: Two types of data integration between geographic objects and geo-tagged social media.

\subsubsection{Frequency Based Semantic Annotation}

Once the nearby social media signals are aggregated for each object, we propose two alternative methods to find the ranking functions that measure the likelihood of a signal relevant to the given geographic object: frequency based methods and probability based method.

Boundary represented geographic objects normally have a larger extent compared to point based objects. Intuitively, a term that occurs frequently within a place may be a relevant annotation. We can count the occurrences of nearby words based on the Term Frequency (TF).

To reduce noisy terms, we can improve frequency based method by smoothing it with a weighting factor, using Inverse Document Frequency (IDF). IDF can measure how much information the word provides by checking whether the word is common or rare across all documents.

\subsubsection{Probability Based Semantic Annotation}

For point based objects, one issue is that aggregating nearby words requires a distance threshold. How to choose such threshold is challenging as different places have different scales of neighborhoods. To address these limitations, we propose to use probability based method which models the relevancy versus the distance. We take the Kernel Density Estimation (KDE) based methods for the ranking problem. KDE has been previously used for modeling human location[7] and generating semantic annotations for mobility data[12]. This work focuses on modeling geo-tagged words with KDE for annotating point based geographic objects. Other than frequency based methods, KDE models the spatial density of the word occurrences and then weights differently for words with different distances.

\section{FREQUENCY BASED SEMANTIC ANNO- TATION}

We start with term frequency $(T F)$ based approach to evaluate the term relevance for annotations based on frequencies of occurrences, and then refine it with document corpus based weighting $(T F-I D F)$ to reduce weights for terms across multiple documents. As multiple occurrences of a term from distinct tweets contribute more than those from a single tweet, we further propose to consider collective tweet weighting ( TF-per-tweet-IDF). Last, since distinct users tend to provide more independent opinions, we introduce collective user weighting (TF-per-user-IDF).

\subsection{Term Frequency Based Weighting}

Given a geographic object, one intuitive semantic annotation method is to rank the nearby social media signals according to the frequencies of occurrence, i.e., term frequency $(\mathbf{T F})$. Formally, given a geographic boundary $b_{i}$ or a geographic point $p_{i}$, a containment based query contain $\left(b_{i}, D\right)$ and a range-within-distance query $\operatorname{range}\left(p_{i}, D, \delta\right)$ aggregate all the social media documents located within the boundary of $b_{i}$ as $D_{b_{i}}$ and documents within the range of a distance $\delta$ to $p_{i}$ as $D_{p_{i}}$. The TF based ranking function $T F\left(b_{i}, w_{j}\right)$ and $T F\left(p_{i}, w_{j}\right)$ then measures the relevance of a word $w_{j}$ in equation 1 , where $W_{b_{i}}$ and $W_{p_{i}}$ indicate the set of associated features extracted from the document $D_{b_{i}}$ and $D_{p_{I}}$ respectively.

$$
\begin{aligned}
T F\left(b_{i}, w_{j}\right) & =\mid\left\{w_{j} \epsilon W_{b_{i}}: l_{w_{j}} \text { in } L_{b_{i}}\right\} \mid \\
T F\left(p_{i}, w_{j}, \delta\right) & =\left|\left\{w_{j} \epsilon W_{p_{i}}: \operatorname{dist}\left(l_{p_{i}}, l_{w_{j}}\right)<\delta\right\}\right|,
\end{aligned}
$$

The TF based ranking function does not distinguish common words, stop words, or expression words, such as "im", "start", and "time", which overwhelm important terms with richer semantics. To filter such non-relevant words, we use the algorithm of term frequency-inverse document frequency (TF-IDF) to smooth the term frequencies.

\subsection{Document Corpus Based Weighting}

Given a large collection of documents, TF-IDF is used to represent the relative importance or uniqueness of a term to a specified document. Intuitively, TF-IDF based method gives a low weight to a word that is frequent in one document but also appears across many other documents. In our scenarios, tweets are usually short in length but accumulate exponentially as regard to the total number of documents, 
which provide a rich data source for smoothing the term frequencies.

Given a geo-tagged social media corpus $D$ and a geographic object $b_{i}$ or $p_{i}$, the TF-IDF based ranking function $\operatorname{TFIDF}\left(b_{i}, w_{j}, D\right)$ and $\operatorname{TFIDF}\left(p_{i}, w_{j}, D\right)$ measure the relevance of a word $w_{j}$ in equation 2 , where $W_{b_{i}}$ and $W_{p_{i}}$ indicate the set of associated features extracted from the document $D_{b_{i}}$ and $D_{p_{I}}$ respectively.

$$
\begin{aligned}
\operatorname{TFIDF}\left(b_{i}, w_{j}, D\right) & =T F\left(b_{i}, w_{j}\right) * I D F\left(D, w_{j}\right) \\
I D F\left(D, w_{j}\right) & =\log \left[\frac{N_{D}}{1+\left|\left\{d_{k} \epsilon D: w_{j} \epsilon W_{d_{k}}\right\}\right|}\right] \\
\operatorname{TFIDF}\left(p_{i}, w_{j}, \delta, D\right) & =T F\left(p_{i}, w_{j}, \delta\right) * I D F\left(D, w_{j}\right) \\
I D F\left(D, w_{j}\right) & =\log \left[\frac{N_{D}}{1+\left|\left\{d_{k} \epsilon D: w_{j} \epsilon W_{p_{k}}\right\}\right|}\right],
\end{aligned}
$$

\subsection{Collective Tweet Weighting}

The collective signals from overall social media context have been effectively utilized to smooth a term frequency through the weight of inverse document frequency. Our spatial data integration framework, on the other hand, generates a local context through the aggregated nearby documents, which contain additional knowledge for the relevance of a term w.r.t. targeted objects. For example, a term mentioned in multiple tweets in the local context should imply higher relevance than a term with multiple occurrences coming from a single tweet.

We propose collective tweet weighting (TF-per-tweetIDF) to smooth the direct term frequency by counting term occurrences per tweet, i.e., multiple mentions in a single tweet count only once. Formally, the collective weighting method is defined in equation 3 , where $D_{b_{i}}$ is aggregated documents located within the boundary of $b_{i}$, and $D_{p_{i}}$ is aggregated documents within the range of a distance $\delta$ to $p_{i}$.

$$
\begin{aligned}
T F_{\text {tweet }}\left(b_{i}, w_{j}\right) & =\left|\left\{d_{k} \epsilon D_{b_{i}}: w_{j} \epsilon W_{d_{k}}\right\}\right| \\
T F_{\text {tweet }}\left(p_{i}, w_{j}, \delta\right) & =\left|\left\{d_{k} \in D_{p_{i}}: w_{j} \epsilon W_{d_{k}}\right\}\right| \\
T F_{\text {tweet }} I D F\left(b_{i}, w_{j}, D\right) & =T F_{\text {tweet }}\left(b_{i}, w_{j}\right) * I D F\left(D, w_{j}\right) \\
T F_{\text {tweet }} I D F\left(p_{i}, w_{j}, \delta, D\right) & =T F_{\text {tweet }}\left(p_{i}, w_{j}, \delta\right) * I D F\left(D, w_{j}\right),
\end{aligned}
$$

\subsection{Collective User Weighting}

One added knowledge within the social media platform is the author information. Terms from the same user tend to be similar and different users tend to generate more independent contents. By identifying the original source of each term, we can distinguish terms coming from the same user or from diverse users.

We propose collective user weighting (TF-per-user-IDF): the multiple occurrences of a term from the same user will be counted only once and the frequency of a term from distinct users will be the count of distinct users. Formally, it is defined in equation 4 , where $U_{b_{i}}$ and $U_{p_{i}}$ are the set of users who generate the social media documents in $D_{b_{i}}$ and $D_{p_{i}}$ respectively. $W_{u_{k}}$ indicates the set of associated features extracted from the document of the user $u_{k} \in U_{b_{i}}$ or $u_{k} \in U_{p_{i}}$.

$$
\begin{aligned}
T F_{u s e r}\left(b_{i}, w_{j}\right) & =\left|\left\{u_{k} \epsilon U_{b_{i}}: w_{j} \epsilon W_{u_{k}}\right\}\right| \\
T F_{u s e r}\left(p_{i}, w_{j}, \delta\right) & =\left|\left\{u_{k} \epsilon U_{p_{i}}: w_{j} \epsilon W_{u_{k}}\right\}\right| \\
T F_{u s e r} I D F\left(b_{i}, w_{j}, D\right) & =T F_{u s e r}\left(b_{i}, w_{j}\right) * I D F\left(D, w_{j}\right) \\
T F_{u s e r} I D F\left(p_{i}, w_{j}, \delta, D\right) & =T F_{u s e r}\left(p_{i}, w_{j}, \delta\right) * I D F\left(D, w_{j}\right),
\end{aligned}
$$

Discussion. The frequency based methods with weighting are based on the assumption that the spatial relevance of a tweet to an OSM object is certain, i.e., a tweet is clearly contained in the object boundary. Thus, such methods work better for large objects with boundary based representations.

\section{PROBABILITY BASED SEMANTIC AN- NOTATION}

For point based object representation, to associate spatial relevance of a tweet to an OSM object, a circle based approximate buffer is created for spatial matching. Choosing the right threshold for the buffer is challenging as each type of places may have very different scale of neighborhood. For example, a coffee shop has a much smaller extent than a church. A popular landmark such as a tourism attraction around a coffee shop may generate many tweets which are irrelevant to the shop. A frequency based method is not working any more, as it treats all nearby words with same spatial relevance regardless of the distance.

For objects with only point based representations, or for objects with very small extents, the spatial relevance will be dependent on the distance between the tweet and the object. We propose a probability based method to model the probability of the relevance of a word to a geospatial object as a function of the distance. KDE is a non-parametric method for estimating a density function from a random sample of data. Prior work has utilized KDE for modeling the spatial density of word occurrences, or individual mobility data[12]. Our work investigates KDE model for annotating geographic points with the spatial probability of word occurrences.

\subsection{Kernel Density Estimation}

As mentioned earlier, frequency based method for boundary objects without enough spatial extents leads to data sparsity problem and introduces more noise from nearby landmarks. The essence of KDE based model is to estimate a spatial density from word occurrences. The counts of word occurrences are then smoothed out with the spatial density over the continuous space.

Formally, let $\mathbf{L}^{\mathbf{w}_{\mathbf{j}}}=\left\{l_{1}^{w_{j}}, l_{2}^{w_{j}}, \ldots, l_{N}^{w_{j}}\right\}$ refer to all occurrences of a word $w_{j} \epsilon V_{D}$ where $V_{D}$ is from a geo-tagged social media corpus $D$. Given a two-dimensional Gaussian kernel function $\mathbf{G}$ and a fixed bandwidth $\mathbf{h}$, we propose a ranking function (KDE-fixed) for the word $w_{j}$ w.r.t. a geographic point $p_{i}$ as described in equation 5 , where $C_{h}$ refers to a $2 \times 2$ covariance matrix.

$$
\begin{aligned}
K D E_{\text {fixed }}\left(p_{i}, L^{w_{j}}, G, h\right) & =\frac{1}{\left|L^{w_{j}}\right|} \sum_{k=1}^{N^{w_{j}}} G_{h}\left(l_{k}^{w_{j}}, l_{p_{i}}\right) \\
G_{h}\left(l_{k}^{w_{j}}, l_{p_{i}}\right) & =\frac{1}{2 \pi h} \exp \left[-\frac{1}{2}\left(l_{k}^{w_{j}}, l_{p_{i}}\right)^{T} \mathbf{C}_{\mathbf{h}}^{-\mathbf{1}}\left(l_{k}^{w_{j}}, l_{p_{i}}\right)\right] \\
C_{h} & =\left[\begin{array}{ll}
h & 0 \\
0 & h
\end{array}\right],
\end{aligned}
$$

Similar to the idea of collective tweet weighting, the KDEfixed method refer to the word occurrences per tweet, i.e., multiple mentions of a word in a single tweet count only once. To extend the KDE-fixed method with collective user weighting, we propose an alternative KDE-fixed method (KDEfixed-per-user): the multiple occurrences of a word from the same user will be counted only once and the centroid 
point of these multiple occurrences will present the location of the word.

With the KDE-fixed method, each word occurrence contributes to the overall ranking score according to its distance to the targeted point, which provides a more accurate estimation about the relevance and omits the requirement of a boundary for encompassing nearby words. Previous work $[10,7]$ suggests that the choice of the bandwidth value $h$ determines the shape of the resulting spatial density. While a smaller $h$ produces a sharper peaked distribution around the locations of word occurrences, an inappropriately large bandwidth $h$ would generate an oversmoothed estimation. In the experiment, we try to adjust $h$ with different values for our datasets with different types of objects.

\subsection{KDE with Adaptive Bandwidth}

The above KDE-fixed method requires tuning of the bandwidth which is time consuming. Besides, the smoothing is homogeneous for all the words regardless of the difference of their spatial densities. For example, the name of an iconic symbol in the city tend to accumulate near the landmark address. The bandwidth in such situation should obviously be different with that around a sparsely populated area.

In order to prevent either overfitting or oversmoothing, we take another adaptive based approach (KDE-adaptive) where the bandwidth is set adaptively for the KDE based ranking function. Given a term $w_{j} \epsilon V_{D}$, a customized bandwidth $h$ would be generated according to the provided occurrence locations $L^{w_{j}}$. Inspired by Breiman et al. [4], we set the bandwidth $h_{w_{j}}$ as the distance between the targeted geographic point $p_{i}$ and its $k$-th nearest neighbor. The formal definition of KDE-adaptive is described in equation 6 , where $h_{j}$ refers to the Euclidean distance to the $k$-th nearest neighbor to $l_{p_{i}}$.

$$
\begin{aligned}
K D E_{\text {adatpive }}\left(p_{i}, L^{w_{j}}, G\right) & =\frac{1}{\left|L^{w_{j}}\right|} \sum_{k=1}^{N^{w_{j}}} G_{h_{j}}\left(l_{k}^{w_{j}}, l_{p_{i}}\right) \\
C_{h_{j}} & =\left[\begin{array}{cc}
h_{j} & 0 \\
0 & h_{j}
\end{array}\right],
\end{aligned}
$$

Similar to the KDE-fixed method, multiple mentions of a word in a single tweet count only once. For the alternative KDE-adaptive method with collective user weighting (KDE-adaptive-per-user ), the multiple occurrences of a word from the same user count once and use the centroid point as its location.

In our problem setting, noisy signals such as stop words, expression words or spams accumulate across time could overwhelm the spatial semantics of our interest. By setting the bandwidth according to $k$-th nearest neighbor, the adaptive kernel approach tunes the bandwidth inversely with the word density. For the word with a low density, the distance of its $k$-th nearest neighbor to a given object is larger than the word with a dense occurrence, which results in a larger bandwidth to adapt the sparseness of the data. In our experiment, we evaluate different choices of $k$ for the datasets.

\section{EXPERIMENTAL EVALUATION}

We evaluate the performance of our method to annotate multiple types of geospatial objects extracted from UK with tweets. We also compare the difference between frequency based method and probability based method for annotating point based geographical objects. We provide both groundtruth based comparison and case studies with manual evaluation.

\subsection{Datasets}

We downloaded the entire OSM data and filtered the data to generate a collection of representative places from UK. The places of interests are selected according to the OSM tags. The geo-tagged tweets corpus was collected for the period between Nov 1, 2014 and Sep 09, 2015 and contains $343,779,205$ geo-tagged tweets in total. For simplicity, only English words from tweet contents are considered as annotation candidates in the experiments. The overall statistics of the datasets is summarized in Table 1.

\begin{tabular}{c|c|c}
\hline \multicolumn{2}{c|}{ Geospatial Data Sources } & \# of Objects \\
\hline OSM & Boundary Objects & $4,156,607$ \\
\cline { 2 - 3 } in UK Area & Point Objects & 506,086 \\
\hline \multicolumn{2}{c}{ Geo-tagged Tweets } \\
$11 / 01 / 2014-09 / 09 / 2015$ & $343,779,205$ \\
\hline
\end{tabular}

Table 1: Description on datasets

\subsection{Experimental Settings}

Name detection experiment. We design a name detection experiment to assess our proposed semantic annotation methods. While it is difficult to provide ground-truth to evaluate semantic annotations, one special OSM tag, the name for a given place, is provided by most OSM objects and could serve as ground truth to evaluate our proposed annotation methods. We build a ground truth dataset by extracting a subset of places with their name tags contained in OSM data and appearing in the nearby tweet contents.

\begin{tabular}{c|c|c}
\hline Geographic Format & Place Category & \# of Objects \\
\hline \multirow{4}{*}{ Boundary Objects } & Station & 365 \\
\cline { 2 - 3 } & Tourism & 362 \\
\cline { 2 - 3 } & Church & 1,914 \\
\cline { 2 - 3 } & Stadium & 487 \\
\cline { 2 - 3 } & Park & 9,329 \\
\cline { 2 - 3 } & Theatre & 386 \\
\cline { 2 - 3 } & Shop & 447 \\
\hline \multirow{4}{*}{ Point Objects } & Station & 2,467 \\
\cline { 2 - 3 } & Church & 1,317 \\
\cline { 2 - 3 } & Tourism & 749 \\
\cline { 2 - 3 } & Sport & 571 \\
\hline
\end{tabular}

Table 2: Statistics of Ground Truth Datasets

Ground truth Dataset Generation. To build the ground truth dataset, we first cross-matches the whole geotagged tweets corpus with geographical objects in OSM. For boundary based objects, the integrated corpus includes all boundaries that contain at least one tweet. For point based objects, the integrated corpus includes all points with at least one tweet detected within their buffered circle ranges. In our experiment, the radius distance for the buffered circle is set up to 0.002 decimal degrees (worth up to 250 meters). We then filter out the ground truth corpus with the place names appearing in the nearby tweet contents. A set of representative place categories is used in the following experiments (Table 2).

Evaluation Metrics. Given a geographic object, the result is a sorted list of relevant words ordered by their ranking scores from a semantic annotation method. We validate whether the top $\mathrm{K}$ words with the highest ranking scores will contain the place name. Given a collection of boundary based objects or point based objects in the ground truth, the name detection accuracy is the percentage of places with their names contained in the top $\mathrm{K}$ annotations. 


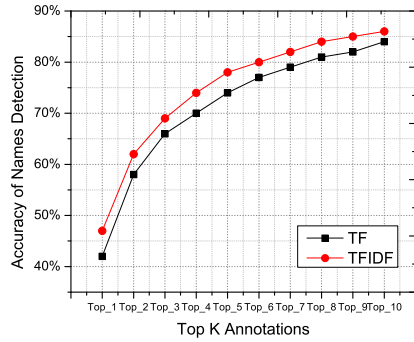

(a)

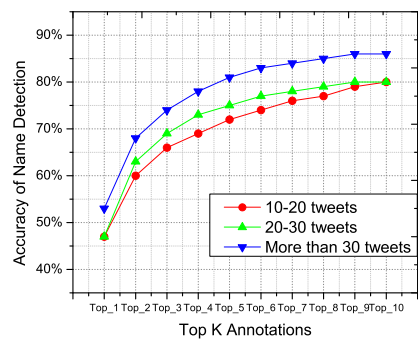

(c)

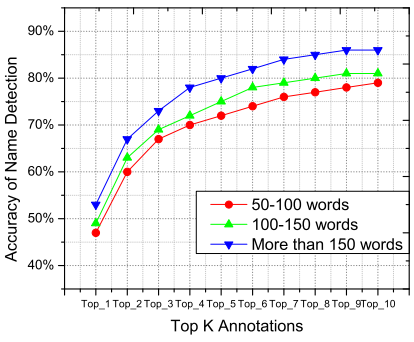

(b)

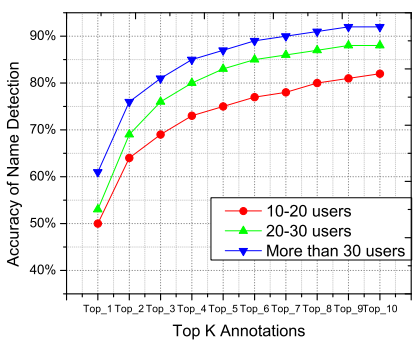

$(d)$

Figure 3: (a) Name detection accuracy of TF and TF-IDF methods for top K results; (b)-(d) Name detection accuracy of TF-IDF method with boundary object grouping based on user count, tweet count and word count respectively.

\subsection{Evaluation of Frequency Based Methods}

We evaluate frequency based methods for boundary based objects with place name detection. Figure 3(a) illustrates the performance of TF and TF-IDF methods on all seven types of boundary based objects in the ground truth datasets. TF-IDF clearly outperforms TF for name detection accuracy. Such result indicates that collective signals from overall social media context can effectively smooth the direct term frequency through weighting in IDF.

In reality, some areas are more densely populated than others. We further examine the performance of TF-IDF method on places with different popularity. We then group all boundary based objects according to their contained user counts, tweet counts and word counts respectively. As shown in Figure 3(b)-(d), places that contain more signals tend to have a higher accuracy for name detection experiments, no matter how the places are grouped. With a closer examination, however, we find that grouping with user counts has a higher improvement than grouping with word couts and tweet counts. This implies that higher user appearance can supply richer information for annotations.

Based on the observation, we design two variants of TFIDF method discussed in section 3, i.e., TF-per-tweet-IDF, and TF-per-user-IDF, which incorporate local signals from aggregated nearby documents. We then use four frequency based methods to annotate names of the places containing tweets coming from at least 30 users. The results in Figure 4(a) demonstrate the effectiveness of user information for enhancing annotations, and indicate that a larger number of distinct users mentioning the same keyword will provide stronger evidence for the relevance of the keyword to the corresponding places.

We also evaluate the performance for different place cate-

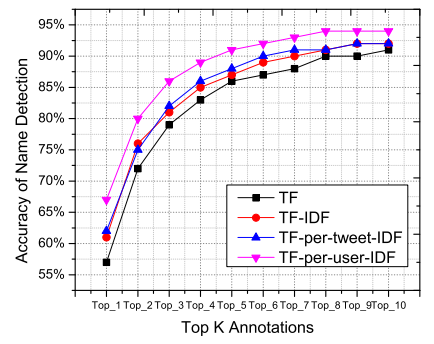

(a)

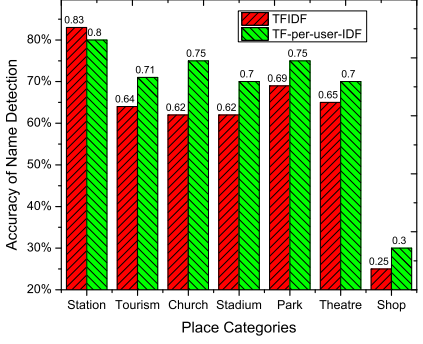

(b)
Figure 4: (a) Name detection accuracy of frequency based methods; (b) Name detection accuracy of TFIDF and TF-per-user-IDF for different place categories.

gories. Figure 4(b) shows the name detection accuracy using top 10 annotations for different place categories. TF-peruser-IDF consistently outperforms TF-IDF across almost all categories. The only exception is railway stations, where TF-IDF performs better. Besides, the overall accuracy for railway stations is also much higher than other categories. This implies that geo-tagged tweets from stations contain more spatial dependent information and have less noise. On the other hand, the overall accuracy for shops has a very low accuracy, which implies its nearby social context has a much lower "signal to noise ratio".

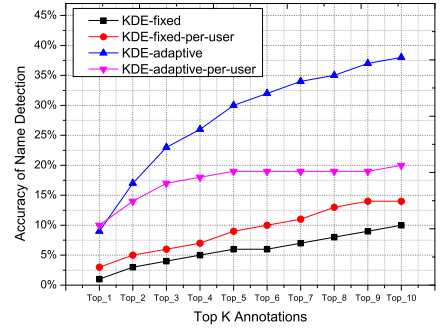

(a)

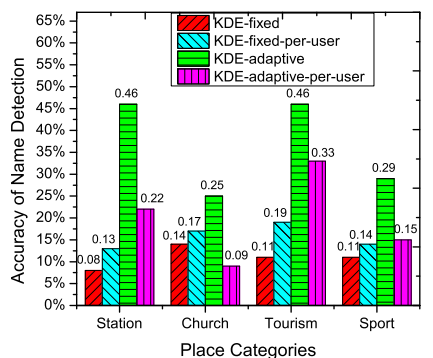

(b)
Figure 5: (a) Name detection accuracy of probability based methods; (b) Name detection accuracy of KDE-fixed-with-weighting and KDE-adaptive for different place categories.

\subsection{Evaluation of Probability Based Methods}

We evaluate probability based methods for annotating geographical points. We first compare different KDE based methods. Figure 5(a) shows the name detection accuracy of all point objects combined. Figure 5(b) shows the accuracy with top 10 annotations for different types of places. We experiment different parameters and compare the highest accuracies for both KDE-fixed based methods (with bandwidth value $h$ set as 0.0001 decimal degree) and KDE-adaptive based methods (with the number of neighbors as 2). The adaptive bandwidth methods clearly outperform fixed bandwidth methods.

The probability based methods rely on the bandwidth parameter $h$ for estimating the word density distribution. In order to prevent either overfitting or oversmoothing, smaller bandwidth values should be assigned to denser words and 


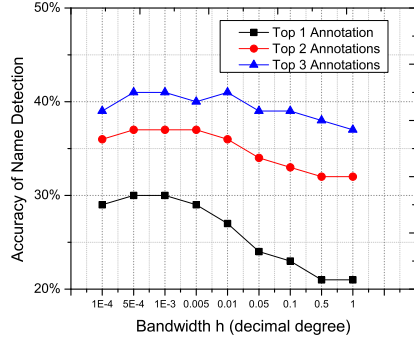

(a)

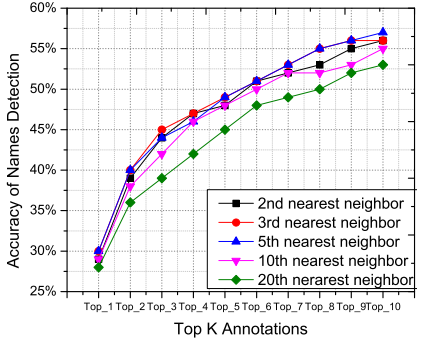

(b)
Figure 6: (a) Name detection accuracy with increasing bandwidth $h$ for KDE-fixed; (b) Name detection accuracy for KDE-adaptive with $h$ set to the distance between the place and its k-th nearest geotagged tweet.

larger ones should fit to sparse words. To better understand the influence of bandwidth, we study the effect of bandwidth on accuracy. Figure 6 illustrates the accuracy trend with varying bandwidth for detecting names of churches. We experiment on churches because this category of places (in our dataset) includes both tourism hotspots with many dense words and local churches with only sparse words. We observe that KDE-fixed reaches the highest accuracy with $h$ between 0.0005 and 0.01 decimal degrees $(20$ meters to 1 kilometer). For KDE-adaptive, we find that the accuracy is decreased when $h$ is larger than the distance between the place and its 10 th nearest neighbor.

\subsection{Frequency Based Methods vs Probability Based Methods}

We then compare frequency based methods and probability based methods for detecting the names of point based objects. For frequency based method, an approximate buffer with a distance threshold $\delta$ is used to identify nearby tweets. The results in Figure 7(a) show that, when the range search threshold $\delta$ is decreased, the number of places with their names detected from nearby tweets is also decreased. This suggests that a smaller distance threshold $\delta$ will lead to a loss of relevant information.

We compare the performance of frequency based methods versus probability based for identifying names of churches. As shown in Figure 7(b), both KDE-fixed (with $h$ set to 0.01 decimal degree) and KDE-adaptive (with the number of neighbors as 3) outperform all frequency based methods.

\subsection{Case Studies}

We also perform two case studies to evaluate the annotation results with human interpretation. We first classify annotations into three categories: explicitly relevant, implicitly relevant, and non-relevant. Explicitly relevant annotations are about major characteristics of an object, for example, the name and theme of a museum. Implicitly relevant annotations are more about derived information or minor information, for example, a collection in a museum.

\subsubsection{Boundary Object: Imperial War Museum North}

For the boundary based object (Imperial War Museum North), we compare top 20 annotations from four frequency based methods. The existing tags in OSM (Figure 8(a)) mainly contain the name and place category. Example ex-

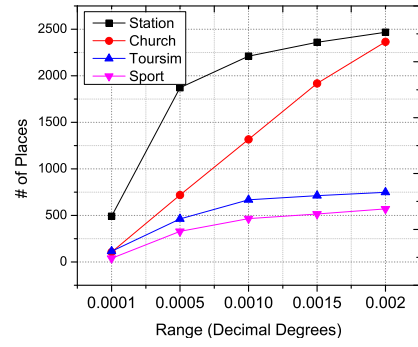

(a)

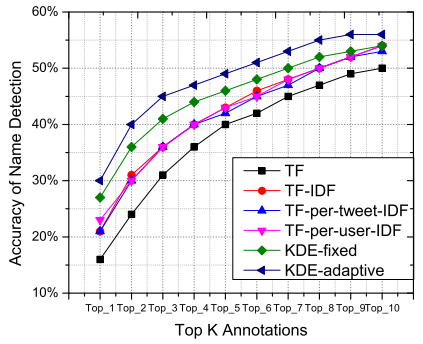

(b)
Figure 7: (a) Number of places with names detected from nearby tweets with varying range search threshold $\delta$; (b) Name detection accuracy of different semantic annotation methods for point based objects.

plicitly relevant annotations include "war", "museum", and "iwmn" (the abbreviation for the full name). Implicitly annotations include "architecture" 4 , "wellingtonbomber" (hashtag for wellington bomber), "gunturret" (hashtag for gun turret), which are either the collections or the characteristics of Imperial War Museum. Non-relevant words include names of nearby places such as "univeristyofmanchester" (hashtag for University of Manchester) or the city name alone 'manchester' which is too abroad as an annotation. As shown in Figure 8(b), TF-per-user-IDF produces more relevant annotations (6 explicitly relevant and 4 implicitly relevant) than other frequency based methods.

\subsubsection{Point Object: Tower Bridge}

For the point based object (Tower Bridge), we compare top 20 annotations from two frequency based methods and two probability based methods. Explicitly relevant annotations include "walkway" or "glasswalkway" (the hashtag for glass walk way), which are either famous exhibition or a feature of Tower Bridge. The non-relevant words include common language or names of nearby businesses and landmarks. As shown in Figure 9(b), non-relevant words from frequency based methods contain more common language, and probability based methods generate names of nearby landmarks. KDE-adaptive method produces more relevant annotations than KDE-fixed method. The KDE-adaptive method in this case study detects one explicit relevant word as the top 1 result and 2 other implicitly relevant words among the top 6 results.

\section{RELATED WORK}

While the data consumption of OSM mainly comes from map rendering, geocoding, and smart routing, its analytical value has yet to be explored. The previous OSM data analytical work mainly focuses on the measurement of content bias [8] or predictive analysis such as fine-grained population estimation[3]. In this work, we integrate OSM data with geo-tagged social media for semantic annotation. Recently, Wu et al.[12] use geo-tagged tweets to annotate Twitter users. Work from Sengstock et al. in [9] extracts latent geographic features from Flickr tags, which is for general

\footnotetext{
4 The two original tweets for "architecture" are: \#imperialwarmuseumnorth \#manchester \#salfordquays ... Impressive architecture \#lovemanchester https://t.co/eS4tfRkEq \#manchester https://t.co/SVndM4ARCk
} 
(a) A Boundary Object with its OSM Tags

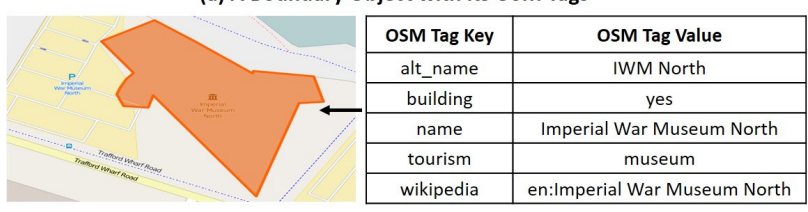

(b) Top 20 Annotations by Frequency Based Methods

\begin{tabular}{|c|c|c|c|}
\hline TF & TF-IDF & TF-per-tweet-IDF & \begin{tabular}{|l|} 
TF-per-user-IDF \\
\end{tabular} \\
\hline war $^{1}$ & imperial $^{1}$ & imperial $^{1}$ & imperial $^{1}$ \\
\hline museum $^{1}$ & war $^{1}$ & war $^{1}$ & war $^{1}$ \\
\hline imperial $^{1}$ & museum $^{1}$ & museum $^{1}$ & museum $^{1}$ \\
\hline north $^{1}$ & north $^{1}$ & north $^{1}$ & north $^{1}$ \\
\hline manchester & salford & salford & salford \\
\hline salford & $\begin{array}{c}\text { MrBaizen } \\
\text { OnEuropeTour }\end{array}$ & $\begin{array}{c}\text { Mrbaizen } \\
\text { Oneuropetour }\end{array}$ & manchester \\
\hline greater & manchester & manchester & SalfordQuays \\
\hline $\begin{array}{c}\text { MrBaizen } \\
\text { OnEuropeTour }\end{array}$ & SalfordQuays & SalfordQuays & greater \\
\hline SalfordQuays & greater & greater & $i^{1} m^{1}$ \\
\hline posted & $\mathrm{iwm}^{1}$ & $\mathrm{iwm}^{1}$ & mediacity \\
\hline im & travel & travel & posted \\
\hline travel & mediacity & mediacity & im \\
\hline photo & posted & posted & photo \\
\hline food & \begin{tabular}{|c|} 
Imperial \\
WarMuseumManchester
\end{tabular} & $\begin{array}{c}\text { Imperial } \\
\text { WarMuseumManchester }{ }^{2}\end{array}$ & umbrella \\
\hline mediacity & $\mathrm{im}$ & $\mathrm{im}$ & quay \\
\hline iwm & UniversityOfManchester & UniversityOfManchester & architecture $^{2}$ \\
\hline quay & nom & uom & $\begin{array}{c}\text { Wellington } \\
\text { Bomber }^{2}\end{array}$ \\
\hline UniversityOfManchester & photo & photo & GunTurret $^{2}$ \\
\hline \begin{tabular}{c|} 
Imperial \\
WarMuseumManchester \\
\end{tabular} & umbrella & umbrella & julandhur \\
\hline ball & quay & quay & iwmn $^{1}$ \\
\hline
\end{tabular}

(c) Manual Evaluation for the Relevance of Annotation Words

\begin{tabular}{|c|c|}
\hline Relevance & Annotation Words \\
\hline${ }^{2}$ Explicitly relevant & $\begin{array}{c}\text { 'war', 'museum', 'imperial', 'north', 'iwm', } \\
\text { 'ImperialWarMuseumManchester', 'iwmn' }\end{array}$ \\
\hline${ }^{2}$ Implicitly relevant & 'architecture', 'WellingtonBomber', 'GunTurret' \\
\hline NOT relevant & $\begin{array}{c}\text { All other words are not relevant, e.g., the city name 'manchester' (where } \\
\text { the museum is located) is too general to be a relevant annotation. }\end{array}$ \\
\hline
\end{tabular}

Figure 8: Interpretation and evaluation of tweets based semantic annotations for Imperial War Museum North (boundary based geographical object).

geographic knowledge discovery. As to geosocial networking, previous studies that bring together social media users and geographic objects mainly rely on check-in data from Location-based social networks (LBSNs). Most prior works analyze geo-tagged social media within geographic granularity up to street level [6]. In our work, we explore geo-tagged tweets with fine-grained geographic context and extract semantic annotations for individual places of interests.

\section{CONCLUSION}

Vast amounts of spatial big data are being increasingly generated through VGI and social media. Integrating multiple sources of spatial big data could provide new insights and create new forms of value. In this paper, we present integrative spatial data analytics to support geo-tagged tweets based annotation for OSM objects. We first propose frequency based methods optimized through various weighting schemes to annotate objects with clear boundaries, and then propose probability based methods based on KDE optimized with adaptive bandwidth to annotate objects with point based representations. Our experiments from groundtruth comparison and human interpretation of annotation results demonstrate promising results.

\section{Acknowledgments}

This work is supported in part by NSF ACI 1443054 and by NSF IIS 1350885 . (a) A Point Object with its OSM Tags

\begin{tabular}{|c|c|c|}
\hline OSM Tag Key & OSM Tag Value \\
\hline historic & monument \\
\hline name & Tower Bridge \\
\hline & tourism & attraction \\
\hline wikipedia & en:Tower Bridge \\
\hline
\end{tabular}

(b) Top 20 Annotations by Both Frequency Based and Probability Based Methods

\begin{tabular}{|c|c|c|c|}
\hline $\mathrm{TF}$ & TF-per-user-IDF & KDE-fixed & KDE-adaptive \\
\hline bridge $^{1}$ & tower $^{1}$ & LondonRiviera & TowerBridge $^{1}$ \\
\hline tower $^{1}$ & bridge $^{1}$ & TowerOfLondon & StKatharineDocks \\
\hline Iondon & Iondon & TowerBridge $^{1}$ & TheScoop \\
\hline TowerBridge $^{1}$ & TowerBridge $^{1}$ & DesignMuseum & GlassWalkWay $^{2}$ \\
\hline photo & greater & MoreLondon & GlassFloor $^{2}$ \\
\hline greater & thames & TowerHill & artigram \\
\hline $\mathrm{im}$ & $\mathrm{im}$ & brigde $^{1}$ & katharines \\
\hline posted & posted & 20fenChurchStreet & fowd \\
\hline day & photo & TheGherkin & bermondsey \\
\hline uk & uk & WalkieTalkie & wihs \\
\hline thames & day & LondonBridge & LondonRiviera \\
\hline england & WalkWay ${ }^{2}$ & ShardView & MoreLondon \\
\hline city & view & Bermondsey & SuperYacht \\
\hline morning & england & TheShard & CityHall \\
\hline view & tourist & CityHall & TheGherkin \\
\hline night & londres & shard & LondonBridge \\
\hline love & city & gherkin & CheeseGrater \\
\hline time & morning & bflofaniko & Tamise \\
\hline beautiful & river & CheeseGrater & WalkieTalkie \\
\hline londres & travel & ThePetCoach & RenzoPiano \\
\hline
\end{tabular}

\begin{tabular}{|c|c|}
\hline \multicolumn{2}{|c|}{ (c) Manual Evaluation for the Relevance of Annotation Words } \\
\hline Relevance & Annotation Words \\
\hline${ }^{1}$ Explicitly relevant & 'bridge', 'tower', 'TowerBridge' \\
\hline${ }^{2}$ Implicitly relevant & 'WalkWay', 'GlassWalkWay', 'GlassFloor' \\
\hline NOT relevant & $\begin{array}{c}\text { All other words are not relevant, e.g., 'TowerOfLondo' and 'LondonBridge' } \\
\text { are two nearby landmarks with similar names to Tower Bridge. }\end{array}$ \\
\hline
\end{tabular}

Figure 9: Interpretation and evaluation of tweets based semantic annotations for London Bridge (point based geographical object).

\section{REFERENCES}

[1] A. Aji, X. Sun, H. Vo, Q. Liu, R. Lee, X. Zhang, J. Saltz, and F. Wang. Demonstration of hadoop-gis: A spatial data warehousing system over mapreduce. In SIGSPATIAL/GIS, 2013.

[2] A. Aji, F. Wang, H. Vo, R. Lee, Q. Liu, X. Zhang, and J. Saltz. Hadoop-GIS: A High Performance Spatial Data Warehousing System over MapReduce. Proc. VLDB Endow., 2013.

[3] H. Bast, S. Storandt, and S. Weidner. Fine-grained population estimation. In SIGSPATIAL/GIS, 2015.

[4] L. Breiman, W. Meisel, and E. Purcell. Variable kernel estimates of multivariate densities. Technometrics, 1977.

[5] M. F. Goodchild. Citizens as sensors: the world of volunteered geography. GeoJournal, 2007.

[6] R. Lee, S. Wakamiya, and K. Sumiya. Urban area characterization based on crowd behavioral lifelogs over twitter. Personal and ubiquitous computing, 2013.

[7] M. Lichman and P. Smyth. Modeling human location data with mixtures of kernel densities. In SIGKDD, 2014.

[8] G. Quattrone, L. Capra, and P. De Meo. There's no such thing as the perfect map: Quantifying bias in spatial crowd-sourcing datasets. In $C S C W, 2015$.

[9] C. Sengstock and M. Gertz. Latent geographic feature extraction from social media. In SIGSPATIAL/GIS, 2012.

[10] B. W. Silverman. Density estimation for statistics and data analysis. CRC press, 1986.

[11] H. Vo, A. Aji, and F. Wang. Sato: A spatial data partitioning framework for scalable query processing. In SIGSPATIAL/GIS, 2014.

[12] F. Wu, Z. Li, W.-C. Lee, H. Wang, and Z. Huang. Semantic annotaion of mobility data using social media. In $W W W$, 2015. 